Paolo De Maina*

ORCID: 0000-0003-3632-494X

Roma, Italy

\title{
Sociological imagination and social promotion: the category of "Agapic Action", Salerno (Italia), 7-8 giugno 2018
}

Il 7 e 8 giugno scorso si è svolto in Italia il Congresso "Sociological imagination and social promotion: the category of 'Agapic Action' to interpret the changes and to imagine new futures".

Promosso dal Research Network Social-One e dall'Università degli Studi di Salerno, il congresso - che voleva essere sia un punto di arrivo che di ri-partenza circa l'approfondimento dell'Agape, oggetto di studio e di approfondimento da vari anni per Social One - si è svolto in partenariato con 12 università e Enti di Ricerca nel mondo.

Il research network "Social-One, scienze sociali in dialogo," nasce nel 1999 in Italia. È un gruppo internazionale di sociologi e studiosi del Servizio Sociale che da quasi 20 anni è attivo nell'ambito della ricerca sociale. Qual è la mission del gruppo? Contribuire, attraverso gli strumenti epistemologici, metodologici, teorici e di ricerca tipici delle scienze sociali, alla costruzione di un mondo più unito. Questo significa innanzitutto fare della sociologia una

* Paolo De Maina, PhD, specialista in Programmazione e Gestione dei servizi e delle politiche sociali, membro di Social-One, comunità scientifica internazionale di sociologi e studiosi del servizio sociale, membro del Centro interdisciplinare di studi Scuola Abbà, collabora con la rivista Città Nuova. Indirizzo: Via Piave, 15, 00046 Grotaferrata (Rm) - Italia; mail: p.demaina@libero.it 
disciplina al servizio della società e in grado di mettere in luce il protagonismo degli attori sociali.

La spinta per la nascita di questo gruppo è stata rappresentata da un evento per noi molto significativo: siamo nel 1996 e Chiara Lubich, una donna laica cattolica, fondatrice del Movimento dei Focolari e vincitrice nel 1986 del premio Unesco per l'educazione alla pace, riceve il dottorato honoris causa all'Università di Lublino in Polonia in Scienze Sociali. La motivazione per la quale le viene conferito questo titolo è significativa:

aver avuto il merito di cominciare una rivoluzione copernicana nelle scienze sociali, per aver promosso, con la vita e con il pensiero, il dialogo nei diversi campi, in ambito interreligioso, interculturale e sociale come fattore chiave per la costruzione e il mantenimento della pace.

In quanto sociologi e studiosi del servizio sociale ci sentiamo chiamati in causa per primi da questa motivazione, intravvedendo in essa una possibilità di riflessione sociologica, specialmente in risposta alle sfide del pluralismo di oggi. Così nel 1999 abbiamo costituito il nostro gruppo.

Tornando al Congresso di Salerno, più di 100 studiosi e appassionati di temi sociali provenienti da tre Continenti si sono trovati per discutere, presentare proposte e scambiare buone pratiche su 4 temi: pluralità e dialogo, protagonismo comunitario e cultura della condivisione, cooperazione e politiche sociali. Il terreno comune di incontro è stato l'engagement delle Scienze Sociali alla ricerca di un nuovo "pluriversalismo", ovvero "universalismo a più voci" in cui, accanto alla promozione dei diritti umani, siano riconosciute anche le diversità culturali e il protagonismo delle comunità locali e dei molteplici attori sociali, in un'ottica di condivisione, interdipendenza e partecipazione.

Sono stati circa 70 gli studiosi che hanno risposto al call for paper.

Tra i relatori, numerosi gli studiosi e docenti universitari, fra cui: Gennaro Iorio e Raffaele Rauty dell'Università di Salerno; Paulo Henrique Martins dell'Ufpe di Recife; Alberto Merler, Università di Sassari; Ortega Santo Pedro José, Università di Santo Domingo; Eduarda Rocha e Yvana Carla Fechine de Brito, UFPE Recife; Mauro Magatti, Emanuela Mora e Michele Colasanto, Università Cattolica del Sacro Cuore di Milano; Izabela Wagner, Università of Varsavia, William A. Calvo-Quiros, Università del Michigan USA; Anna Maria Campanini, Università Bicocca Milano; Luigi Gui, Università di Trieste; Marcelo Salas, USAL, Buenos Aires - Argentina; Rudina 
Rama, Università di Tirana - Albania; Silvia Cataldi Università La Sapienza di Roma.

Gennaro Iorio, ordinario presso l'Università di Salerno, nella relazione introduttiva ha sottolineato che

Nel nostro percorso di elaborazione e approfondimento del concetto di amore-agape abbiamo avuto modo di interfacciarci con studiosi del calibro di Axel Honneth, Michael Burowoy, Luc Boltanski, Alain Caillè, Edgar Morin, Margaret Archer, Paulo Henrique Martins, solo per citare i non italiani. L'incontro con questi studiosi ci ha aperto nuove prospettive di lavoro sul concetto di definizione dell'agape secondo una prospettiva sociologica, volta a rendere operativo un concetto sul piano sostantivo, euristico e empirico. Oggi dobbiamo dare inizio ad una nuova fase. Il momento è quello di lavorare al concetto di agape per rendere la sociologia e le scienze del servizio sociale realmente al servizio della promozione sociale. Lavoriamo con la consapevolezza di fare un lavoro "inattuale", nel senso inteso da Nietzsche, cioè "un pensiero critico, che collide, rompe disturba gli argomenti ricorrenti, e il modo di pensare convenzionale" (Nietzsche, 1997).

Il sociologo brasiliano Paulo Henrique Martins, professore all'Università Federale di Pernambuco di Recife e presidente di ALAS Associazione Latinoamericana di Sociologia, nella sua relazione tra il resto ha detto:

Un tema fondamentale che emerge dalla discussione in questo Congresso è quello del "bene comune", che stimola l'arte di vivere insieme e il principio di una "comune umanità" che prende in considerazione quattro punti: comune umanità, socialità comuni, l'individuazione e l'opposizione controllata con attenzione al morale, politico, ecologico e alle condizioni economiche [...]. Questo aiuta a capire che ci sono soluzioni pratiche al processo caotico della colonizzazione planetaria e che essa richiede nuove pratiche di azione 'agapica'. In questa prospettiva, il convivialismo non può presentarsi come un discorso unificato, ma come una narrazione da tradurre in diverse situazioni ispirate da un atto amorevole e solidale e rispondente a vari immaginari sociali e culturali anti-coloniali.

Accanto ai momenti in plenaria, si sono svolte Sessioni parallele sia per la presentazione dei call paper sia per approfondimenti tematici. Importante l'ambientazione, in quanto l'Università di Salerno è un vero e proprio Cam- 
pus Universitario che offre spazi fruibili e ampi che consentono una maggiore socializzazione in tutti i momenti del congresso.

Interessante l'attenzione ad un ancoraggio empirico. Infatti accanto alla riflessione scientifica, il Convegno ha anche lanciato un SOCIAL EXPO per la condivisione di best practices in ambito sociale cui hanno partecipato circa 20 diverse Associazioni europee e dell'America Latina.

Gli atti del Congresso intitolati Sociological Imagination and Social Promotion, ed. Paolo Contini and Maria Rosalba Demartis (ISBN 978889 5697062) sono presentati alla pagina web della Social-One: http://social-one. org/images/Atti/ATTI.pdf 